\title{
PERFILES
}

\section{David Montgomery (1927-2011)}

\author{
Ludmila Scheinkman
}

El pasado 2 de diciembre fallecía, a los 84 años de edad, el historiador David Montgomery, eminente representante de la New Labor History norteamericana. El presente artículo se propone analizar su trayectoria político-intelectual así como sus principales trabajos, señalando aquellos aspectos de su obra, escasamente difundida en nuestro país, que consideramos de vigencia, actualidad y relevancia tanto en lo metodológico, como para reflexionar sobre la agenda de investigación. Junto a sus planteos e hipótesis específicas sobre la historia de los trabajadores y el movimiento obrero norteamericano de fines de siglo XIX y principios del XX, pretendemos destacar aquí la envergadura de su empresa teórico-intelectual y politica.

\section{Los años en la fábrica y en el PC}

Nacido en 1927 en Bryn Mawr, Pennsylvania, Montgomery sirvió en el Cuerpo de Ingenieros del Ejército durante la Segunda Guerra Mundial. Asistió luego a Swarthmore College en Pensilvania donde se graduó en 1950 con los más altos honores con un título de pregrado en Ciencias Políticas. Como él mismo señala en una entrevista, refiriéndose a sus años de juventud, no provenía de una familia de izquierda pero siempre tuvo un interés político, razón por la cual estudió ciencias políticas con la idea de que alli podría aprender "algunas formas en las que se pudiera transformar este mundo. Desde la infancia, me había acosado la antigua pregunta: ¿por qué los que trabajan más duro son los que menos ganan?” (Buhle y Naison, 1980: 47). ${ }^{1}$ Sin embargo, lejos de con-

1. Las traducciones de toda la bibliografia en inglés son nuestras. Una versión de esta entrevista fue publicada en una compilación por The Radical Historians Organisation (MAHRO), véase Abelove et al. (1984). 
tinuar una carrera académica, los años 50 lo encuentran trabajando como maquinista, destacándose como activista en la organización de base en las fábricas. Se involucró en la actividad sindical como miembro activo del Sindicato de Trabajadores Eléctricos Unidos y la Asociación Internacional de Maquinistas y ocupó numerosos cargos gremiales, entre ellos delegado sindical, miembro del comité legislativo y miembro del comité ejecutivo local.

También fue por estos años, en 1951, que ingresó en el Partido Comunista (PC) de los Estados Unidos, si bien era una época de decadencia y conflicto interno en el seno del mismo. Las posiciones del PC sobre asuntos internacionales, justicia racial y sindicalismo lo impulsaron a integrar sus filas, en las que encontró un espacio político desde donde mantener una actividad vinculada al trabajo cotidiano a nivel de la fábrica, para intervenir y lograr una influencia sobre los trabajadores, más allá de las políticas del partido (Buhle y Naison, 1980: 39), si bien serían estas últimas las que lo llevarian a distanciarse.

El historiador Jon Wiener, en el obituario que escribio para The Nation, señala que fue la fuerte represión macartista la que llevaria a Montgomery a abandonar las fábricas y dedicarse a la historia. Tras las persecuciones del FBI y los despidos sufridos, "Finalmente (...) el único trabajo que pude conseguir era en un taller donde sólo trabajaba un obrero. Lo incorporé a la unión, pero en este punto me di cuenta que me habian derrotado, así que renuncié y me convertí en historiador" (Wiener, 2011). Fue así que trabajando de maquinista se encontró cada vez más interesado en la historia (Buhle y Naison, 1980: 47).

Esto coincide también con su alejamiento del PC, junto a muchos otros militantes que rompen en el congreso partidario de 1957, a raíz de la invasión a Hungría del año anterior, pero también por un descreimiento ideológico, coincidente con la decadencia del papel y la influencia del partido entre los trabajadores. Si bien este proceso fue análogo al que experimentaban por esos años en Inglaterra los historiadores vinculados al PC británico, en Estados Unidos, por el contrario, aquellos que se alejaron del partido no continuaron ligados como grupo con publicaciones o elaboraciones teóricas en común, sino que siguieron derroteros individuales (Buhle y Naison, 1980: 42). Al igual que sucedió con muchos de sus pares norteamericanos y británicos, la etapa más rica y creativa de su trabajo intelectual comienza a partir de su alejamiento del PC, ya que la vida intelectual oficial del mismo era "sofocante".

Pero a diferencia de otros decepcionados con el PC, Montgomery se mantuvo en el marco de la izquierda, incluso frente a los embates del posmodernismo y el giro lingüístico. La defensa de los trabajadores como sujeto histórico de transformación, la vigencia de la clase como categoria explicativa de la realidad, el papel del socialismo en esta trans- 
formación y la necesidad de la intervención política se sumaron a su acción militante contra la guerra y el armamento nuclear, a los talleres de historia en los sindicatos y a su participación y apoyo en las huelgas universitarias. En suma, mantuvo una amalgama entre compromiso político y trabajo erudito.

Tanto la experiencia en el comunismo como su trabajo en las fábricas influyeron en la elección de su objeto de estudio y su interés por indagar el radicalismo en el movimiento obrero, aspectos que van a recorrer el conjunto de su carrera académica.

Fue así que, expulsado de las fábricas, regresó a la universidad, donde obtuvo su doctorado en historia en la Universidad de Minnesota en 1962. Ejerció como docente 14 años en la Universidad de Pittsburgh y luego en Yale a partir de 1979, habiendo recibido premios a la enseñanza en las dos instituciones. En ambas ciudades siguió apoyando activamente a los sindicatos locales y fue un orador frecuente y participante activo en las movilizaciones contra la guerra de Vietnam. Su tesis doctoral fue la base del primero de sus libros y de más de 85 artículos y capítulos de libros que publicó tanto solo como en coautoría, a la vez que se destacó formando e incentivando a estudiantes y futuros historiadores.

\section{De la historia del movimiento sindical a la historia de los trabajadores: la New Labor History}

Los años 60 verán en la historiografia norteamericana, como parte de un movimiento más amplio, el ascenso de las ciencias sociales y el surgimiento de una historia social que se propondrá como alternativa a la "vieja" historia politica. Mientras que una de sus vertientes se volcó fuertemente hacia la cuantificación y la historia serial, la que nos interesa aquí estuvo más bien inspirada por los desarrollos de los historiadores marxistas británicos y los annalistes ocupados en la historia de las mentalidades.

La labor history comenzó en los Estados Unidos a inicios del siglo XX de la mano de economistas que impulsaron el estudio de las relaciones laborales en el contexto de la profesionalización de la ciencia económica. Particularmente influyentes fueron los trabajos de John R. Commons y su joven colega, Selig Perlman, quienes entre otros conformaron la "Escuela de Wisconsin". Ésta se dedicó al estudio de la negociación colectiva y las organizaciones sindicales, es decir a una historia de las instituciones del movimiento obrero. A partir de esto, comenzó a elaborarse una interpretación donde las organizaciones norteamericanas, a diferencia de sus pares europeas, radicales y politizadas, eran vistas como conservadoras y economicistas, orientadas a atemperar los aspectos más duros del capitalismo más que a combatir al sistema en sí. 
Estos sindicatos tenian una "conciencia laboral" (job-consciousness), pero no una conciencia "socialista", puesto que rechazaban el marxismo (Norrell, 1990). Implícita en el fondo de estas preocupaciones, estaba una respuesta a la vieja pregunta del sociólogo alemán Werner Sombart de 1905: “¿por qué no hay socialismo en los Estados Unidos?”.

Frente a esta historiografia es que una generación, la primera de historiadores propiamente dichos, va a proponer en los '60 una "nueva" labor history, centrada no ya en el estudio del "movimiento sindical" sino de los "trabajadores". El cambio no era tan sólo de énfasis. En un artículo seminal, David Brody, uno de sus principales referentes, señalaba que uno de los puntos aglutinantes de la corriente era su crítica a una historia puramente institucional, centrada en el estudio de las organizaciones pero que no tenía en cuenta también a los obreros mismos. (Brody, 1979: 111). Los análisis multi-causales, la interdisciplinariedad y el acercamiento a las ciencias sociales permitieron estos enfoques novedosos, y una explosión de trabajos en el área. El énfasis en el estudio del radicalismo de los trabajadores y la identificación con las eras de insurgencia del pasado (Fink, 1991: 420) llevaron, asimismo, a cuestionar muchos de los presupuestos sobre el "anti-radicalismo" del movimiento obrero norteamericano dando una respuesta novedosa a la paradoja de Sombart, reinterpretando el significado político de las luchas de los siglos XIX y XX y haciendo énfasis en el radicalismo y valores de la cultura obrera (Kimeldorf y Stepan-Norris, 1992: 513).

La New Labor History pasó a ocupar un lugar central en la renovación de la disciplina histórica de la mano de la historia social, plasmada en el acceso de sus historiadores a cargos centrales en las instituciones académicas y la apertura de departamentos dedicados al tema.

Brody identifica dos influjos poderosos de los que se nutrió la corriente. Uno fue político: la radicalización de los años 60 y la vinculación de muchos de estos jóvenes historiadores con la Nueva Izquierda. ${ }^{2}$ Este movimiento operó un desplazamiento en la importancia central otorgada por la izquierda al movimiento obrero, para centrarse en un sujeto disperso -los "nuevos movimientos sociales"- y en iniciativas "contraculturales". De hecho una línea historiográfica dominante en la New Labor History va a ser la trazada por Herbert Gutman, con un enfoque fuertemente cultural que, en lugar de ampliar la mirada, en muchos casos simplemente dejó de lado el estudio de la política y las

2. Esta corriente estuvo conformada mayormente por estudiantes universitarios blancos más o menos radicales o liberales, unidos en la defensa de la democracia, los derechos civiles, los movimientos de reforma universitaria y las protestas contra la Guerra de Vietnam. Conformaban una organización "suelta", dispersa y sin un programa político aglutinante, por oposición a la "vieja izquierda" partidaria. 
organizaciones sindicales. Ésta no fue, no obstante, la línea de Montgomery y otros historiadores, como David Brody y Melvyn Dubofsky, que mantuvieron el foco y el interés en las organizaciones del movimiento obrero. En el caso de Montgomery, su interés por el estudio de los trabajadores se vincula a su ya señalado paso por el PC. Fue crítico de la Nueva Izquierda por su indiferencia hacia el movimiento obrero y su rechazo al papel de los liderazgos políticos (Buhle y Naison, 1980: 51).

En segundo lugar, Brody detalla el fuerte impacto de la obra del historiador británico Edward P. Thompson y del grupo de historiadores vinculados inicialmente al PC británico, con su innovadora propuesta metodológica y la apertura de una nueva agenda de indagación. Ésta se hace sentir fuertemente en el ensayo pionero de Herbet Gutman (1976), "Work, Culture and Society in Industrializing America", uno de los primeros intentos por trascender los limites de la historia sindical (Brody, 1979:111).

Dubofsky (1977) señala también la influencia de la escuela francesa de los Annales y su interés por el rol activo de la "gente común" en la historia, por las mentalidades populares, los rituales y el comportamiento colectivo.

Pero debemos destacar que hay elementos particulares que distinguen a la tradición norteamericana. La dirección que estaba tomando la historiografia en otros campos de estudio, en una búsqueda por recuperar la agencia de los hombres y mujeres que habian sido pasados por alto por la historiografia anterior, influyó fuertemente: "La historiografia americana en general estaba «descubriendo" a las mujeres, los negros y las minorías étnicas" (Norrell, 1990: 231), en un dinámica que no puede dejar de vincularse con el auge de los movimientos feministas y por los derechos de los negros. Por eso una de las características de la New Labor History va a ser su mayor sensibilidad por los distintos clivajes identitarios que influyeron en la conformación de la clase obrera y sus organizaciones.

Este cruce entre clase, género y etnicidad cobra gran relevancia en la obra de David Montgomery. Los estudios de la comunidad, como en el trabajo pionero de Gutman y el énfasis en los análisis de los trabajadores de base y sus luchas por el "control obrero" en el lugar de trabajo, impulsados por Montgomery, son, según Brody (1979), otras de las particularidades que distinguen a la New Labor History. Sus historiadores se preocuparon por dar cuenta de una realidad más compleja, pero tratando de no caer en la fragmentación en tópicos excluyentes (historia urbana, historia de la familia, historia de las mujeres, etc.) como fue común en la "nueva historia" social, sino buscando conservar "la unidad que es esencial para entender la naturaleza humana" (Dubofsky, 1977: 250). 
Sin embargo, este peligro estaba presente sobre todo en los enfoques culturales que relegaron la experiencia laboral para centrarse en la comunidad y la cultura: el de caer en una serie de historias particulares -de inmigrantes, de negros, de mujeres, de la comunidad, etc.- que en última instancia serian la historia de aquellos factores que dividieron a la clase obrera (Dubofsky, 1981: 245). Esta línea, exacerbada, lleva implícita una pregunta de fuertes implicancias metodológicas: vistas las múltiples y profundas líneas que dividieron a los trabajadores, ¿'es posible seguir hablando de clase obrera? ¿Qué es lo que les dio unidad? Aquí es que nuevamente la experiencia laboral en el lugar de trabajo, las huelgas, la política y los sindicatos entran en el relato. Stanley Aronowitz ha señalado que, si bien el capital divide a los trabajadores de muchas maneras ("cualificados" y "no cualificados", "intelectuales" y "manuales", raza, género y sexualidad), una de las intervenciones cruciales del sindicalismo de masas fue insistir en la unidad basada en los intereses comunes de aquellos forzados a trabajar por un salario (1990: 172).

\section{La obra de Montgomery}

Los trabajos de Montgomery se orientaron fundamentalmente a reconceptualizar la historia de la clase obrera norteamericana durante el siglo XIX y principios del XX. En su primer libro, Beyond Equality: Labor and the Radical Republicans, 1862-1872, de 1967, aún no se habian hecho sentir los influjos renovadores de la historia social. Se trata de un análisis que combina la historia politica con la historia del movimiento obrero, en un intento de aprehender el papel de los trabajadores en la escena nacional moderna, en vinculación con las tensiones raciales y regionales. Alli explora el movimiento obrero durante la etapa de la Reconstrucción posterior a la Guerra Civil y sus efectos sobre los alineamientos políticos del período, con el objetivo de reinterpretar al Republicanismo Radical a partir de este prisma novedoso. A raíz de esto concluye que el avance y desarrollo de la organización obrera y la capacidad de negociación colectiva de los trabajadores conllevó una crisis para los radicales y sus principios, como se muestra en el ejemplo de la lucha por la jornada de ocho horas. El planteo de igualdad ante la ley en una nación unificada no era suficiente para los trabajadores movilizados, quienes, en sus demandas, fueron "más allá de la igualdad". "Fue en el conflicto de clases, en otras palabras, donde los sueños radicales encallaron y naufragaron" (Montgomery, 1967: X).

Diversos aspectos de esta tesis han sido discutidos. Varios autores han marcado que se trata de un planteo sumamente aventurado y original, para el que falta, sin embargo, cierto sustento empírico, ya que el grueso del libro se dedica al movimiento obrero, pero no profundiza en 
el análisis de los "republicanos radicales": quiénes eran, cuáles eran sus principios e ideas respecto de los trabajadores, etc. (Mandel, 1968; Cox, 1968; Current, 1968). No obstante, se destaca la validez metodológica de una aproximación que pretendió integrar a los trabajadores en el escenario mayor de la politica. Como señala Montgomery en la entrevista a la Radical History Review: "Si bien mi especialidad es la historia de la clase obrera, el tema que intento abordar es la historia del capitalismo (...) Beyond Equality se centró en la etapa de la Reconstrucción porque esa época me ofrecía una entrada para aprehender el impacto de los trabajadores norteamericanos en las corrientes principales de la vida politica nacional. Éste ha sido mi interés central desde entonces" (Buhle y Naisson, 1980: 48).

Montgomery se colocará ya en la órbita de la historia social a partir de su estadía en Inglaterra. Entre 1967 y 1969 enseñó en la Universidad de Warwick, donde colaboró con E. P. Thompson para establecer el Centro para el Estudio de Historia Social, y fue profesor de historia de Norteamérica entre 1986 y 1987 en Oxford. Viró entonces su interés hacia el ascenso y caída de la militancia laboral a fines de siglo XIX y principios del XX. En Workers' Control in America (1979) analizó cómo distintos grupos de trabajadores industriales cualificados -obreros metalúrgicos y mineros, entre otros- trataron de "controlar" la naturaleza y ritmos laborales, y cómo su poder en el lugar de trabajo fue gradualmente erosionado por la mecanización y la introducción de sistemas burocratizados de administración en las fábricas. Esta obra, uno de sus trabajos más importantes, reviste especial importancia para los lectores locales ya que se halla traducida al español, editada en 1985 bajo el título El control obrero en Estados Unidos: estudios sobre la historia del trabajo, la tecnología y las luchas obreras. Montgomery se centra aquí en los conflictos y las luchas -a veces silenciosas y veladas, otras abiertas, como en las huelgas-de los trabajadores con tradiciones de oficio a fines del siglo XIX frente a los embates de los empresarios. Los trabajadores cualificados habrian enfrentado al individualismo burgués, consumista y competitivo con un "código moral" igualitario.

Esta hipótesis ha sido discutida entre otros por Dubofsky (1981) quien ha planteado el interrogante de cómo ponderar hasta qué punto este código moral era mayoritario entre los trabajadores, puesto que en su mayoria no se habian incorporado a las uniones y sindicatos donde este ideal "colectivista" habría primado. Crítica similar realiza el autor respecto de la "resistencia al capitalismo" entre los inmigrantes. ¿No habrian estado, en su mayoría, más preocupados por aumentar sus salarios y adquirir propiedades (particularmente viviendas)? (Ver también Patterson, 1982: 744 y Perlman, 1980: 658).

La influencia de la obra de Harry Braverman, con su atención al 
proceso de trabajo y el análisis del espacio laboral, sale a la luz cuando Montgomery analiza la inserción, a principios del siglo XX, de los trabajadores inmigrantes -e incluso las mujeres- en los trabajos menos cualificados y más mecanizados. Los intentos patronales de quebrar un estilo de vida esencialmente rural para conformar una fuerza de trabajo capaz y disciplinada muestran que ésta, no obstante, desarrolló también una tendencia a la huelga, una resistencia al trabajo y una actitud poco "cooperativa" con la patronal. El autor trabaja luego la década del 20 , cuando se logra finalmente imponer esta nueva racionalidad en las fábricas y el poder de las organizaciones sindicales disminuye. Los trabajadores, desprotegidos, logran de todos modos expresar a través de distintas estrategias su disconformidad con el sistema fabril. Este orden de cosas se veria transformado sustancialmente a raíz de la depresión económica y el New Deal, que conllevó un importante papel para las acciones de los trabajadores en los 30 .

Montgomery introduce aquí la importancia central de la acción (o inacción) del Estado en las relaciones entre trabajo y capital. La acción coercitiva en los 20, "el último bastión de defensa" del poder de las patronales (Montgomery, 1979: 158), se va a transformar en los 30 para garantizar la emergencia de un sindicalismo estable y seguro en las industrias de producción masiva; esto, en último término, aquietó y desmovilizó al movimiento obrero. Es que a partir del New Deal y en la segunda posguerra el Estado va a aparecer con una fuerte intervención para garantizar la "armonia". El diagnóstico de Montgomery es, de todos modos, optimista: los trabajadores debian tornar a la acción política para defenderse y avanzar en sus intereses. Frente a visiones pesimistas que destacaban el conservadurismo de la clase obrera (Brody, 1993), nuestro autor confiaba, de forma un poco ilusa, en que los impulsos radicales de los trabajadores hacia el control del lugar de trabajo y los sindicatos llevarian a una ruptura con la fórmula del New Deal (Montgomery, 1979: 175). Ruptura que no obstante no se produciria.

The Fall of the House of Labor: The Workplace, the State and American Labor Activism, 1865-1925 es sin duda su obra principal, lamentablemente no traducida al español. Candidato finalista al Premio Pulitzer en la categoría de no ficción en 1989, en este trabajo Montgomery amplía su rango de análisis para incluir no sólo a los trabajadores cualificados, sino también a los operarios, los jornaleros y los trabajadores manuales no cualificados que construyeron los ferrocarriles, los subterráneos y los sistemas de alcantarillado en la época. Se trata también de una obra no tanto de los sindicatos y conflictos laborales, como de los hombres y mujeres en el lugar de trabajo: trabajadores cualificados, masculinos y nativos (particularmente en las industrias del hierro y el acero), jornaleros inmigrantes y afro-americanos, operarias mujeres en la industria 
textil y del vestido. Alli recorre no sólo la experiencia de la American Federation of Labor (AFL) sino las otras "mansiones" o "casas del trabajo" de los obreros norteamericanos (socialistas, católicos y anarquistas) desde 1880 hasta los años 20, cuando con la asistencia del Estado nacional, las patronales lanzaron un feroz asalto contra las prerrogativas de los trabajadores. En la década de 1920, concluye, la América moderna había sido creada por las protestas de sus trabajadores.

Diferentes aspectos de la obra han sido comentados o criticados por varios autores. Leon Fink (1989) señala que Montgomery asume la visión convencional de la victoria de la administración científica y la "pacificación" del movimiento obrero en los años 20, en lugar de explorar las resistencias. William Lazonick (1989), por su parte, ha advertido que, en un esfuerzo por enfrentarse al determinismo tecnológico de muchos trabajos, Montgomery minimiza el impacto del cambio técnico y no tiene en cuenta sus determinantes sociales (las necesidades del capital). Así, reduce las transformaciones en los medios de producción a cambios en la forma de administración del trabajo (la administración científica propuesta por Taylor) sin analizar los cambios en los medios de producción.

Sin embargo, tanto sus críticos como sus defensores han señalado que esta obra es una referencia ineludible para cualquier estudioso del período. En una narrativa compleja, combina una historia social de la experiencia de los trabajadores en el sitio de trabajo con las interacciones y conflictos con las patronales y el estado. Asimismo, busca vincular este análisis con el marco más amplio de las relaciones entre los centros industriales y las periferias, como parte de la teoria del sistema mundo de Immanuel Wallerstein. Se trata de un relato sensible a las diferencias de género, étnicas y nacionales que operaron como factores de diferenciación interna, y en ocasiones como trabas a la unidad de clase. Tal es así cuando recorre la organización sindical en la industria del hierro, regida por un código ético de conducta que, si por un lado planteaba un desafio al control patronal del lugar de trabajo y una defensa del control obrero, por el otro se definía en términos "masculinos", étnicos y raciales, ya que aplicaba al hombre blanco, cualificado y nativo. Aunque la mirada de Montgomery es empática, no omite ni evade los aspectos desagradables del movimiento obrero norteamericano, si bien tampoco los profundiza. Señala, por ejemplo, la resistencia de la Amalgamated Association of Iron and Steel Workers a admitir a los trabajadores negros, finalmente aceptados por su creciente peso numérico (Montgomery, 1987:27), lo que de todos modos no soluciona el problema del racismo. Otro tanto puede decirse de la discriminación a los trabajadores inmigrantes (ubicados en los peores trabajos, menos cualificados y peor pagos) por sus propios pares; éstos se resistian y oponían también al 
ingreso de las mujeres en la industria (ya que a medida que avanzaba la mecanización no era necesaria ni la fuerza ni la destreza), con el argumento de que "robaban" el trabajo a los hombres. De todos modos Montgomery logra mostrar los intentos por superar aquellas divisiones en pos de configurar una unidad de la clase obrera sobre la base de una experiencia común en los lugares de trabajo.

En Citizen Worker..., profundiza la paradoja que, para los trabajadores norteamericanos, significó la ampliación de la ciudadanía política en el siglo XIX (la democracia) junto a la libertad de mercado: lejos de reforzarse mutuamente, fueron en desmedro una de otra. Mientras los trabajadores adquirian derechos cívicos, se restringia su poder colectivo, se constreñía su acción política a la participación electoral y se reforzaba el poder coercitivo policial tanto dentro como fuera del lugar de trabajo.

Ha sido señalado que tanto la fuerza como la debilidad del libro se halla en la poderosa exposición de esta paradoja constitutiva de la economía política de la democracia burguesa. Mientras Olivier Zunz (1995: 399) considera que el relato es unicausal, ya que responsabiliza exclusivamente por esta situación al capitalismo y sus cosificadas y abstractas "fuerzas del mercado" (restando agencia y responsabilidad a los mismos trabajadores por su racismo y sexismo), Bryan D. Palmer considera que se trata de un cuestionamiento "a la vez elegantemente simple y tentador que lo abarca todo" (1995: 234).

\section{Los limites de la New Labor History}

Estos enfoques, con todo lo que han aportado, también han sido objeto de criticas que han señalado algunos de sus límites. La critica "externa" ha provenido de investigadores, tanto de izquierda como de derecha, que no compartían sus presupuestos (Bogue, 1986: 143). En el primer grupo podemos mencionar las criticas a Montgomery por parte de Mark Perlman, economista especializado en el estudio del movimiento obrero y no casualmente hijo de Selig Perlman. Este acusa a Montgomery y a los historiadores de la "nueva" Labor History (a la que considera ni nueva ni renovadora) de "predicadores" que realizan análisis "prescriptivos" y románticos sin la suficiente base empírica y con una evidente "inclinación por la defensa", más que por el análisis (Perlman, 1980). Rowland Berthoff, por su parte, en "Writing a history of things left out", señala que las "nuevas" historias revisionistas, tratando de cubrir los "baches" en los relatos de la historia norteamericana, han trasplantado conceptos e ideas de otros contextos (particularmente, de los historiadores marxistas británicos) con escasa base empírica para sostenerlos y han tenido grandes dificultades en armar un relato coherente con los 
elementos y producciones dispares y disyuntivos que han "traído a la luz". El autor descarta de plano los intentos incoherentes de la "Nueva Izquierda jeffersoniana-anarquista-marxista-maoísta y radical" (Berthoff, 1986: 11) de encontrar un proletariado "marxista" a la europea en norteamerica, ya que "seis décadas de recurrente depresión y desempleo no fueron suficientes para convertirlos [a los trabajadores] a la solución marxista. (...) No sorprende que su historia resista cualquier revisionismo que insista en la lucha de clases" (Berthoff, 1986: 14). La empresa intelectual pasa, al contrario, por reconstruir la ideologia "igualitarista" propiamente norteamericana, dejando de lado los conceptos foráneos y los métodos populistas de la "historia de abajo hacia arriba".

Desde la izquierda, Elizabeth Fox-Genovese y Eugene Genovese han señalado, en 1976, que la fascinación por lo "popular" hizo a estos historiadores perder de vista el problema principal: el del poder político. Lawrence Mc Donnell, por su parte, indicaba que tanto Gutman como Montgomery subestimaban el poder del capital, y Gavin Wright la insuficiente atención prestada a los problemas económicos y el mercado de trabajo. ${ }^{3}$

Por otro lado, podemos destacar la crítica "interna" de los propios New Labor historians. Ellos han sido los primeros en señalar su propia incapacidad de elaborar, a partir de las distintas investigaciones realizadas, una sintesis integral que pudiera reemplazar las grandes narrativas de Commons-Perlman. Si bien este diagnóstico era compartido, las divergencias surgian en torno al carácter de la sintesis: ¿debía tratarse de una "nueva" historia del movimiento obrero, como sostenían Brody y Montgomery, o de una "nueva" historia nacional que los incluyera (Gutman)? ¿Debía esta historia partir de los aspectos económicos (Brody) o de las clases y sus luchas (Montgomery)? ${ }^{4}$ Apartarse de los claros marcos narrativos de la historia sindical conllevaba la complejidad de elaborar dicha sintesis a partir de la miriada de estudios locales y la variabilidad de la experiencia obrera traídas a la luz por esta historiografia (Brody, 1979: 122).

Otra critica importante, frente al peligro de caer en un culturalismo al estilo de Gutman, se refiere a la necesidad de reincorporar al sindicalismo y la política al análisis. Howard Kimeldorf (1991) lo ha puesto de relieve en "Bringing the Unions Back In (Or Why We Need a New Old Labor History)", y Gerald Friedman ha criticado a Gutman por impulsar el estudio de los trabajadores centrándose en las cambiantes actitudes populares pero sin tener en cuenta su involucramiento en

3. Una reseña de este debate se encuentra en Norrell (1990: 232-233).

4. Ronald Schatz (1984) ha sintetizado este debate. 
estructuras sindicales formales u otras instituciones (Friedman, 2001: 228). Peter J. Rachleff ha señalado que Gutman entiende el conflicto de clases como un choque de expectativas y valores, más que un conflicto de intereses materiales (Rachleff, 1989: 187). Si algunos historiadores de la New Labor History han criticado este culturalismo, otros, por el contrario, abogaron por una profundización en el giro cultural (Buhle y Buhle, 1988).

Otro rango de críticas ha provenido de aquellos especialistas en disciplinas particulares que han acusado, alternativamente a uno $u$ otro autor, de no analizar suficientemente los problemas raciales (ver por ejemplo la crítica de Herbert Hill [1988] a Gutman), el género (ver la crítica de Ileen A. DeVault [1995] a Brody), las divisiones dentro de la clase obrera en general (por ejemplo, la reseña de Olivier Zunz [1995] sobre Montgomery), o de soslayar el peso del racismo y el nativismo, "incómodos" para estos investigadores empáticos con su objeto de estudio (Aronowitz, 1990: 181).

Robert J. Norrell (1990) ha marcado la dificultad de conjugar los marcos analíticos de Montgomery-Gutman para los historiadores del siglo XX y en particular la historia reciente, por el contraste entre el quietismo y conservadurismo del movimiento obrero del presente con las luchas "heroicas" del pasado. Asimismo, ha puntualizado el "romanticismo" de enfoque en que han caído muchas veces. Estas y otras críticas muestran que, si el debate en torno a la New Labor History está lejos de cerrarse, tampoco lo está la vigencia de muchas de las líneas de análisis impulsadas bajo su órbita.

\section{Sosteniendo las barricadas}

En una entrevista realizada en 1997 titulada sugestivamente "¿Cuál es la importancia de la clase trabajadora hoy en día?”, Montgomery señalaba categóricamente, en un período de retroceso del marxismo académico en general, que él seguía considerando a la clase obrera como un agente importante de cambio histórico, y de alli la necesidad de estudiarla. Cuando, frente a los embates del paradigma del giro cultural, la crisis de la historia social ya era patente, muchos cientistas sociales abandonaron el barco pasándose a enfoques culturalistas, particularistas, descreídos de las "grandes narrativas", fragmentando y diversificando a los "actores" de la historia en una miriada de categorias sin jerarquías ni preeminencias. Sin embargo, Montgomery defendió el estudio de la clase obrera como agente del cambio histórico, entendiendo su propio destino como ligado a la clase de la que siempre se sintió parte. Como señala en la entrevista a la Radical History Review: "Nunca tuvimos la más mínima sensación de que estuviéramos escribiendo para 
el pueblo trabajador. Nosotros éramos los trabajadores de Minnesota escribiendo sobre nosotros mismos" (Buhle y Naisson, 1980: 46).

Si este solo hecho merece destacarse, queremos señalar asimismo su defensa de la compatibilidad entre el compromiso político y el trabajo erudito, de lo cual su propia obra es ejemplo (Abarca, Elisalde y Pozzi, 1997: 38). Su compromiso militante no decayó cuando se hallaba ya lejos de las fábricas y bien posicionado en la institución académica. Siempre fue un organizador de trabajadores, de grupos de derechos civiles, y se destaca particularmente su apoyo a la huelga de los administrativos de Yale de 1984 por el reconocimiento del sindicato, para quienes operó como líder inspirador (Wiener, 2011). El historiador Eric Foner lo recuerda como lo opuesto al académico sentado en la torre de marfil (Foner, 2011): cuando los trabajadores de la empresa Colt de armas de fuego en New Haven (donde se encuentra la Universidad de Yale) entraron en una huelga prolongada, Montgomery se unió a la línea de piquete. En el año 2000, como presidente de la Organización de Historiadores Americanos, trasladó las sesiones de la reunión anual en St. Louis desde el hotel que era sede de la reunión hacia una universidad local, como un acto de solidaridad con los litigantes negros que estaban demandando a la cadena hotelera por sus prácticas discriminatorias. ${ }^{5}$

No sólo es el compromiso, sino también la obra de Montgomery que consideramos valiosa para una historiografia periférica como la nuestra, en particular para aquellos interesados en la historia social del movimiento obrero y los trabajadores. La búsqueda de un enfoque de totalidad, donde se vincule a los trabajadores con las transformaciones en la industria, la administración, los cambios en la estructura empresarial y la organización patronal, y con el desarrollo global del capitalismo, parece una agenda desafiante y digna de tenerse en cuenta. Más allá de las críticas (y autocriticas) de los New Labor historians a su incapacidad de producir una obra de sintesis, la aspiración a explicar los "grandes problemas" e integrar la historia de los trabajadores en la

5. Esto no debe opacar, sin embargo, el agudo aislamiento de los "radical historians" respecto del movimiento obrero, aspecto que ha sido señalado repetidamente (Aronowitz, 1990; Brody, 1993; Norrell, 1990). Es que, "paradójicamente", la búsqueda de la New Labor History por recuperar los antecedentes del radicalismo y las luchas del movimiento obrero del pasado se daba en un contexto de fuerte quietismo y conservadurismo del movimiento obrero norteamericano. David Brody señala que esta búsqueda se hallaba relacionada, probablemente, con la necesidad de encontrar en el pasado ciertas claves que permitieran a estos historiadores comprometidos con una politica radical "tener algo que decir que pudiera ayudarnos" a solucionar las crisis del presente: por ejemplo, Montgomery desarrollaba sus investigaciones sobre el "control obrero" justamente en oposición a las formas de negociación colectiva de ese entonces (Brody, 1993: 14-17). 
historia más general de la nación y el "sistema mundo" en su conjunto es una preocupación constante en la obra de Montgomery.

Lo mismo puede decirse de la atención prestada al proceso laboral, al análisis del sitio de trabajo y las experiencias "de base", que son también hoy lineas vigentes de trabajo, fructíferas en la historiografia local. La sensibilidad en el enfoque hacia las diferencias de cualificación, raza, género y nacionalidad nos parece por demás fundamental, teniendo en cuenta que se pretende realizar una historia de "los trabajadores", donde se destaca aquello que les dio unidad y cohesionó (aquello que permite identificar y estudiar a este conglomerado humano como un "grupo social", es decir una clase), pero también los obstáculos en ese camino. No se trata de una historia de las mujeres, ni de una historia de los inmigrantes, ni de una historia de los afro-americanos. Como señala Bryan D. Palmer, el autor se sumerge en las texturas de la vida obrera, pero "no va a satisfacer a todos: en su implacable foco en la clase, hay muchos que ven a las mujeres desplazadas, al género dejado de lado con demasiada facilidad, y la raza y la etnicidad acomodadas a las solidaridades del trabajo. Sin embargo, la comprensión de Montgomery es más abierta a estos aspectos de la identidad de clase que lo que sus críticos reconocen, y si su definición de clase tiene sus raíces en el lugar de trabajo, no menosprecia los hogares, calles, barrios y escuelas. Reconoce que todas estas esferas están realmente interconectadas. Y deja hablar a muchas voces en el relato" (Palmer, 1995: 234).

A diferencia de la tradición norteamericana, donde estos aspectos han adquirido grandes desarrollos e incluso una autonomía temática propia, nuestra historiografia local ha sido reacia a incorporarlos, y esto es particularmente así en el caso de los historiadores del movimiento obrero, los trabajadores y las izquierdas. Si en los Estados Unidos, por el vigor de estas tradiciones intelectuales, estos clivajes no podian -ni querian- ser soslayados por la historia obrera, aquí ésta ha sido fuertemente reactiva, y la crítica no ha tenido tal intensidad, siendo ignorada en la mayoría de los casos.

De todos modos, la visión excesivamente empática de Montgomery se torna por momentos condescendiente, con un cierto sesgo populista y una tendencia a ceder a la "clase en sí"; no son menores las criticas que señalan que subestima el peso de las divisiones en la clase obrera norteamericana al diluirlas en las "experiencias de la clase", cuando estas divisiones actuaron (y actúan). El racismo y las diferencias étnicas fueron una política de la clase dominante para dividir a los trabajadores. Es un "fracaso" del sindicalismo norteamericano y de la mayoría de las direcciones de los trabajadores no haber superado en el plano sindical y político la división impuesta en las fábricas.

Asimismo, el peso puesto en el análisis de la experiencia de la clase 
obrera, la vida en las fábricas, etc., no debe opacar la necesidad de explicar la interrelación, profunda hasta la Segunda Guerra, que establecieron los trabajadores con las corrientes politicas de la izquierda, así como el peso de las políticas estatales y patronales para dividir sus filas por medio de la discriminación y el racismo. Y, por lo tanto, los límites de las politicas de las corrientes que influyeron a los trabajadores para superar estas divisiones. Por esto mismo es sintomático que Montgomery no haya abordado en profundidad la influencia del New Deal, cuando la clase obrera se volvió más conservadora, sobre todo después de la Segunda Guerra. Es que, de hecho los trabajadores no "rompieron" con el New Deal, sino con los movimientos que cuestionaron al capital, con las tendencias a la militancia de base y los sit-downs de 1936-1937, hasta el surgimiento del CIO (Congress of Industrial Organizations). Las dos guerras moldearon al movimiento obrero en tanto pusieron a prueba su cooptación al régimen y al estado, y frente a la Segunda Guerra, la prueba no fue superada por las direcciones sindicales. Asimismo, el New Deal sentó las bases materiales para la adhesión de un sector de la clase obrera al "sueño americano" y la exclusión de las amplias masas; de esto se deriva la importancia del análisis de las corrientes que dirigieron a la clase obrera, y cuánto cedieron a este sector.

En un punto, entonces, la "paradoja de Sombart" aún merece ser explicada. La propuesta de Montgomery, a través de las luchas, los valores y la cultura de la clase obrera, debe ser puesta en relación con un análisis de las opciones políticas del movimiento obrero, su relación con los regímenes y con el Estado y los procesos de cooptación de las direcciones sindicales.

Por un lado, la rica tradición de crítica y debate de nuestros pares del norte nos parece de urgente necesidad en el medio local donde abunda la crítica elogiosa y escasea el debate fraterno y fecundo de ideas, posiciones y metodologias. Pero por otro lado nos parece que, si algunos aspectos del proyecto historiográfico de Montgomery tienen en la actualidad una vigencia evidente, otras cuestiones nos quedan planteadas como problemáticas a pensar no sólo desde la clase obrera norteamericana sino para la reflexión en el marco de la historiografia local.

\section{Bibliografia}

Abarca Graciela, Roberto Elisalde y Pablo Pozzi (1997), “¿Cuál es la importancia de la clase trabajadora hoy en día? Entrevista con David Montgomery", Taller. Revista de Cultura, Sociedad y Politica, Vol. 2, n 4, agosto, Buenos Aires: Asociación de Estudios de Cultura y Sociedad, pp. 30-44.

Abelove, Henry, Betsy Blackmar, Peter Dimock y Jonathan Schneer (1984), Visions of History, New York: Pantheon Books-MAHRO. 
Aronowitz, Stanley (1990), “Writing Labor's History”, Social Text, n 25/26: Duke University Press, pp. 171-195.

Berthoff, Rowland (1986), "Writing a History of Things Left Out", Reviews in American History, Vol. 14, $\mathrm{n}^{\circ}$ 1, marzo, The Johns Hopkins University Press, pp. 1-16.

Bogue, Allan G. (1986), "Systematic Revisionism and a Generation of Ferment in American History", Journal of Contemporary History, Vol. 21, ${ }^{\circ}$ 2, abril, Twentieth Anniversary Issue, Sage Publications, pp. 135-162.

Brody, David (1977) "Review of Work, Culture, and Society in Industrializing America: Essays in American Working-Class and Social History by Herbert G. Gutman", The American Historical Review, Vol. 82, $\mathrm{n}^{\circ}$ 1, febrero, The University of Chicago Press, pp. 195-196.

- (1979), "The Old Labor History and the New: In Search of an American Working Class", Labor History, Vol. 20, invierno, Routledge, pp. 11-26.

- (1993), "Reconciling the Old Labor History and the New", The Pacific Historical Review, Vol. 62, $\mathrm{n}^{\circ}$ 1, febrero, University of California Press, pp. 1-18.

Buhle, Mary Jo y Paul Buhle (1988), "The New Labor History at the Cultural Crossroads", The Journal of American History, Vol. 75, $\mathrm{n}^{\circ}$ 1, junio, Organization of American Historians, pp. 151-157.

Buhle, Paul y Mark Naison (1980), "Once Upon A Shop Floor: An Interview With David Montgomery", Radical History Review, Vol. 23, primavera, Washington: Duke University Press, pp. 37-53.

Cox, LaWanda (1968), "Review of Beyond Equality: Labor and the Radical Republicans, 1862-1872 by David Montgomery", The American Historical Review, Vol. 74, $\mathrm{n}^{\circ}$ 1, octubre, The University of Chicago Press, pp. 300-301.

Current, Richard N. (1968), "Review of Beyond Equality: Labor and the Radical Republicans, 1862-1872 by David Montgomery", The Journal of Southern History, Vol. 34, $\mathrm{n}^{\circ}$ 2, mayo, Southern Historical Association, pp. 312-313.

DeVault, Ileen A. (1995), "Review of In Labor's Cause: Main Themes on the History of the American Worker by David Brody", The American Historical Review, Vol. 100, $\mathrm{n}^{\circ} 4$, octubre, The University of Chicago Press, p. 1286.

Dubofsky, Melvyn (1977), "The "New" Labor History: Achievements and Failures", Reviews in American History, Vol. 5, $\mathrm{n}^{\circ}$ 2, junio, The Johns Hopkins University Press, pp. 249-254.

- (1981), "Hold the Fort: The Dynamics of Twentieth-Century American Working Class History", Reviews in American History, Vol. 9, $\mathrm{n}^{\circ}$ 2, junio, The Johns Hopkins University Press, pp. 244-251.

Fink, Leon (1989), "Review of The Fall of the House of Labor: The Workplace, the State, and American Labor Activism, 1865-1925 by David Montgomery", The American Historical Review, Vol. 94, $n^{\circ} 3$, junio, The University of Chicago Press, pp. 881-882. 
- (1991), “«Intellectuals» versus «Workers»: Academic Requirements and the Creation of Labor History", The American Historical Review, Vol. 96, $\mathrm{n}^{\circ}$ 2, abril, The University of Chicago Press, pp. 395-421.

Foner, Eric (2011), "David Montgomery Obituary", The Guardian, December 11, http: / /www.guardian.co.uk/books/2011/dec/11/david-montgomery

Gutman, Herbert G. (1976), Work, Culture and Society in Industrializing America: Essays in American Working-Class and Social History, Nueva York: Kunz.

Friedman, Gerald (2001), "Review of Hard Work: The Making of Labor History by Melvyn Dubofsky", The Journal of Economic History, Vol. 61, n 1, marzo, Cambridge University Press on behalf of the Economic History Association, pp. 227-229.

Hill, Herbert (1988), "Myth-Making as Labor History: Herbert Gutman and the United Mine Workers of America", International Journal of Politics, Culture, and Society, Vol. 2, $\mathrm{n}^{\circ}$ 2, invierno, Springer, pp. 132-200.

Kimeldorf, Howard (1991), "Bringing the Unions Back in (Or Why We Need a New Old labor History)”, con respuestas de Michael Kazin, Alice KesslerHarris, David Montgomery, Bruce Nelson y Daniel Nelson, Labor History, $n^{\circ} 32$, pp. 91-129.

Kimeldorf, Howard y Judith Stepan-Norris (1992), "Historical Studies of Labor Movements in the United States", Annual Review of Sociology, Vol. 18, Annual Reviews, pp. 495-517.

Lazonick, William (1989), "The Breaking of the American Working Class", Reviews in American History, Vol. 17, $\mathrm{n}^{\circ}$ 2, junio, The Johns Hopkins University Press, pp. 272-283.

Mandel, Bernard (1968), "Review of Beyond Equality: Labor and the Radical Republicans, 1862-1872 by David Montgomery", The Journal of American History, Vol. 55, $\mathrm{n}^{\circ} 1$, junio, Organization of American Historians, pp. 154-155.

Montgomery, David (1967), Beyond Equality: Labor and the Radical Republicans, 1862-1872, New York: Knopf.

- (1979), Workers' Control in America: Studies in the History of Work, Technology, and Labor Struggles, New York: Cambridge University Press. [Hay trad. cast.: El control obrero en Estados Unidos: estudios sobre la historia del trabajo, la tecnología y las luchas obreras, Madrid: Ministerio de Trabajo y Seguridad Social, 1985.]

- (1980) “To Study the People: The American Working Class", Labor History, Vol. 21, otoño, Routledge, pp. 485-512.

- (1987), The Fall of the House of Labor: The Workplace, the State and American Labor Activism, 1865-1925, Cambridge University Press.

- (1993), Citizen Worker: The Experience of Workers in the United States with Democracy and the Free Market during the Nineteenth Century, New York: Cambridge University Press. [Hay trad. cast.: El ciudadano trabajador: 
democracia y mercado libre en el siglo XIX norteamericano, México: Instituto Mora, 1993.]

Norrell, Robert J. (1990), “After Thirty Years of 'New' Labour History, There Is Still no Socialism in Reagan Country", The Historical Journal, Vol. 33, $\mathrm{n}^{\circ} 1$, marzo, Cambridge University Press, pp. 227-238.

Painter, Nell Irvin (1989), "The New Labor History and the Historical Moment", International Journal of Politics, Culture, and Society, Vol. 2, $\mathrm{n}^{\circ} 3$, marzo, Springer, pp. 367-370.

Patterson, James T. (1982), "Review of Workers' Control in America: Studies in the History of Work, Technology, and Labor Struggles by David Montgomery", The Journal of Interdisciplinary History, Vol. 12, $\mathrm{n}^{\circ} 4$, primavera, The MIT Press, pp. 743-744.

Palmer, Bryan D. (1995), "Review of Citizen Worker: The Experience of Workers in the United States with Democracy and the Free Market during the Nineteenth Century, by David Montgomery", The Journal of American History, Vol. 82, $\mathrm{n}^{\circ}$ 1, junio, Organization of American Historians, p. 234.

Perlman, Mark (1980), "Review of Workers' Control in America: Studies in the History of Work, Technology, and Labor Struggles by David Montgomery", The Journal of Economic History, Vol. 40, $\mathrm{n}^{\circ} 3$, septiembre, Cambridge University Press, pp. 656-659.

Rachleff, Peter J. (1989), "Two Decades of the «New» Labor History", American Quarterly, Vol. 41, $\mathrm{n}^{\circ} 1$, marzo, The Johns Hopkins University Press, pp. 184-189.

Schatz, Ronald W. (1984), "Labor Historians, Labor Economics, and the Question of Synthesis", The Journal of American History, Vol. 71, $\mathrm{n}^{\circ} 1$, junio, Organization of American Historians, pp. 93-100.

Wiener, Jon (2011), "David Montgomery, 1927-2011", The Nation, December 2, http://www.thenation.com/blog/164954/david-montgomery-19272011

Zunz, Olivier (1995), "Review of Citizen Worker: The Experience of Workers in the United States with Democracy and the Free Market During the Nineteenth Century by David Montgomery", Contemporary Sociology, Vol. 24, no 4, julio, American Sociological Association, pp. 399-400. 